\title{
Microwave-assisted ligand-free, base-free Heck reactions in a task-specific imidazolium ionic liquid
}

\author{
Mahesh G. Dighe and Mariam S. Degani* \\ Department of Pharmaceutical Sciences and Technology, Institute of Chemical Technology, \\ Matunga (E) Mumbai-400019, India \\ E-mail: ms.degani@ictmumbai.edu.in
}

\begin{abstract}
1-(2-cyanoethyl)-3-(2-hydroxyethyl)-1H-imidazol-3-ium tetrafluoroborate (IL-2) in presence of $\mathrm{PdCl}_{2}$ was found to be an efficient and reusable, ligand-free, base-free catalytic system for Heckcoupling of activated and deactivated iodo- and bromoarenes with different olefins. Under microwave irradiation, it exhibited good efficiency in terms of activity, selectivity and recyclability for six consecutive runs without significant loss of activity.
\end{abstract}

Keywords: Task specific ionic liquid, ligand free, Heck reaction, palladium catalyst, microwave irradiation

\section{Introduction}

Heck reaction, a powerful method for synthesizing diverse olefinic compounds, generally involves carbon-carbon coupling of aryl halides with olefins. ${ }^{1}$ In the last few years, considerable development involving coupling of various pseudo-halides such as aryl triflates, ${ }^{2}$ acid chlorides, ${ }^{3}$ aryl anhydrides, ${ }^{4}$ aryl sulfonyl chlorides, ${ }^{5}$ and aromatic diazonium salts ${ }^{6}$ has been reported. Many drugs and intermediates such as naproxen (Anti-inflammatory agent), prosulfuron (Herbicide) and 2-ethylhexyl-p-methoxy cinnamate (sunscreen agent) are synthesized using the Heck reaction. ${ }^{7}$ The catalytic system for an efficient Heck reaction consists of a palladium source, a ligand, base and solvent. Usually, phosphine containing ligands are used in the Heck reaction, as they play an important role in stabilization and in-situ generation of $\operatorname{Pd}(0)$ species from Pd(II) complexes. Due to the high cost, toxicity and thermal instability of the phosphine containing ligands, ${ }^{8}$ various phosphine free catalytic systems ${ }^{9}$ have been developed. However, most of them showed poor efficiency and reusability in palladium catalyzed Heck reactions. ${ }^{10}$ Hence, ligand free homogeneous catalytic systems ${ }^{11}$ were of great interest for palladium catalyzed Heck reactions. However, in homogeneous systems, it is difficult to overcome the problems associated with catalyst/product separation and catalyst reusability. In this concern, 
various heterogeneous catalyst ${ }^{12}$ systems have been developed by immobilization of palladium catalyst. However these systems are failed to show higher activity compared to homogeneous systems due to extensive palladium leaching. ${ }^{13}$

In the last few decades, ionic liquids have gained great interest in synthetic organic chemistry since they are eco-friendly, nonvolatile, recyclable, and possess good thermal properties. ${ }^{14}$ Ionic liquids can not only serve as a good alternative to volatile toxic, organic solvents as a reaction medium but also can provide mobile support to palladium catalysts. Many ionic liquids are reported to be used as reaction media for the Heck reaction, but most of them are used along with expensive phosphine-ligands. ${ }^{15}$ Hence, the synthesis of functionalized ionic liquids (FILs) through the additional incorporation of the specific pendent groups which could act as ligands and could enhance thermal stability, efficiency and recyclability of the catalyst, are areas of interest. Various reported functionalized imidazolium ionic liquids contain functional groups such as, amine, phosphonite, nitrile, hydroxyl and diols, phosphoryls. Some of the functionalized ionic liquids suffer from drawbacks like air and moisture sensitivity, high palladium loading, difficulties in product isolation, tedious recovery processes and low recyclability. ${ }^{16}$ Diol-functionalized ionic liquids have shown good catalytic efficiency in terms of activity and reusability, when used as a kind of ligand for the palladium catalyzed Heck reaction in DMF. ${ }^{17}$ Hence it would be still enviable to develop simpler, cost effective, reusable, phosphine free catalytic system for palladium catalyzed Heck reaction.

Herein we report on 1-(2-cyanoethyl)-3-(2-hydroxyethyl)-1H-imidazol-3-ium tetrafluoroborate $^{18}$ (IL-2) (Scheme 1), as a simplest phosphine free catalytic system which acts not only as a solvent but also as a ligand and base and offers good activity and recyclability for six consecutive runs without significant loss of activity in the air. In this work, initially the Heck reaction was carried out using iodobenzene and ethyl acrylate with $\mathrm{PdCl}_{2}(2.0 \mathrm{~mol} \%)$ in ionic liquid (IL-2) at $100^{\circ} \mathrm{C}$ for $24 \mathrm{~h}$, which resulted in (E)-ethyl cinnamate in low isolated yield $25 \%$. This was significantly improved on application of microwave heating at $100^{\circ} \mathrm{C}$ for $10 \mathrm{~min}$ (Scheme 2). Microwave heating is reported to accelerate the reaction rate in organic synthesis. ${ }^{19}$ A few microwave-assisted Heck reactions are reported in the ionic liquids; however, some of them used phoshine containing ligands. ${ }^{20-21}$

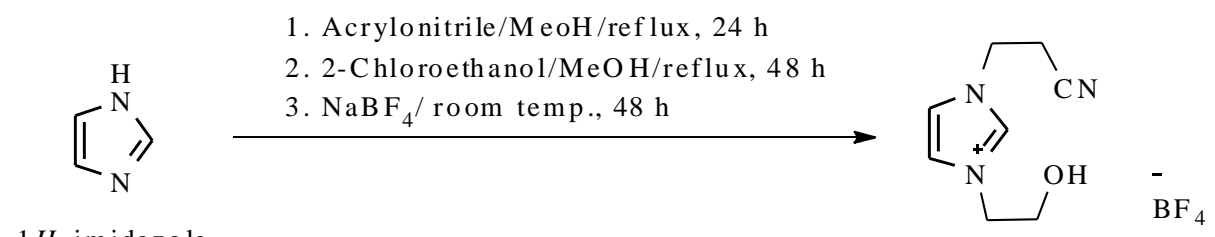

$1 H$-imidazole

( IL - 2)

Scheme 1. Synthesis of ionic liquid (IL-2). 


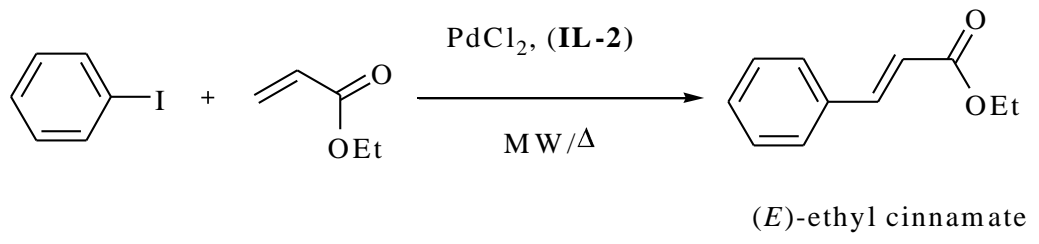

Scheme 2. Heck reaction of iodobenzene with ethyl acrylate in ionic liquid (IL-2).

\section{Results and Discussion}

The reaction conditions were further optimized in terms of palladium loading, reaction temperature and time. $1.0 \mathrm{~mol} \% \mathrm{PdCl}_{2}$ loading resulted in identical yield $69 \%$ as compared to 2.0 $\mathrm{mol} \% \mathrm{PdCl}_{2}$. The rise in temperature from $100{ }^{\circ} \mathrm{C}$ to $120{ }^{\circ} \mathrm{C}$ showed significant increase in isolated yield $81 \%$ (Table 1), however, further increase in temperature to $140{ }^{\circ} \mathrm{C}$, resulted in low yield 64\%. Maximum yield $84 \%$ was achieved, when the reaction was carried out for 5 min. The optimal condition for Heck reaction in ionic liquid (IL-2) was obtained with the following conditions $\mathrm{PdCl}_{2}(0.01 \mathrm{mmol})$, iodobenzene $(1 \mathrm{mmol})$, ethyl acrylate $(2 \mathrm{mmol})$, microwave power $200 \mathrm{~W}$, reaction temperature $120^{\circ} \mathrm{C}$, reaction time $5 \mathrm{~min}$.

Table 1. Effect of different temperature on the yield of Heck reaction

\begin{tabular}{ccc}
\hline Sr. no. & Temperature $\left({ }^{\circ} \mathrm{C}\right)$ & Isolated yield $(\%)$ \\
\hline 1 & 80 & 37 \\
2 & 100 & 69 \\
3 & 120 & 81 \\
4 & 140 & 64 \\
\hline
\end{tabular}

The versatility of the optimized catalytic system for Heck reaction was evaluated with variety of substrates with different electronic and steric effects. The results are summarized in Table 2. Cross-coupling products of different iodoarenes (Table 2, runs 1-7) and bromoarenes (Table 2, runs 8-11) with ethyl acrylate and styrene were obtained in good yields under mild reaction condition $\left(120{ }^{\circ} \mathrm{C}, 5-10 \mathrm{~min}\right)$; attributed to high reactivity of iodo- and bromoarenes. However, due to the steric hindrance, the coupling of 2-bromo methyl benzoate with different olefins (Table 2, runs 12-13) resulted in moderate yield. Good isolated yield were obtained when the effect of the alkyl chain length of terminal vinyl acrylates were studied (Table 2, runs 14-18). However, activated aryl chlorides were unsuccessful to give desired product, when tried to couple with ethyl acrylate under the same reaction condition (Table 2, runs 19-20). 
Table 2. Heck reaction of various aryl halides in ionic liquid (IL-2)

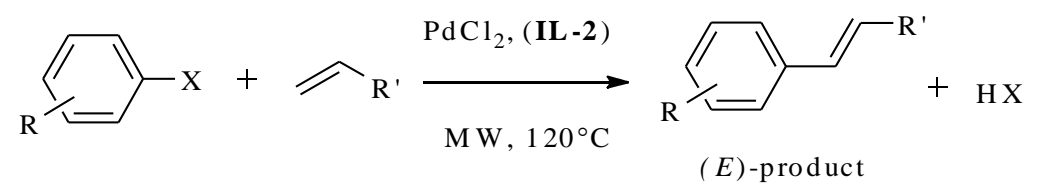

\begin{tabular}{|c|c|c|c|c|c|}
\hline Entry & $\mathrm{R}$ & $\mathrm{X}$ & $\mathrm{R}^{\prime}$ & $\begin{array}{l}\text { Time } \\
(\mathrm{min})\end{array}$ & $\begin{array}{l}\text { Isolated yield } \\
\qquad(\%)^{\mathrm{a}}\end{array}$ \\
\hline 1 & $p-\mathrm{COCH}_{3}$ & I & $-\mathrm{CO}_{2} \mathrm{C}_{2} \mathrm{H}_{5}$ & 5 & 86 \\
\hline 2 & $\mathrm{H}$ & I & $-\mathrm{CO}_{2} \mathrm{C}_{2} \mathrm{H}_{5}$ & 5 & 84 \\
\hline 3 & $p-\mathrm{CO}_{2} \mathrm{C}_{2} \mathrm{H}_{5}$ & I & $-\mathrm{CO}_{2} \mathrm{C}_{2} \mathrm{H}_{5}$ & 5 & 88 \\
\hline 4 & $p-\mathrm{OCH}_{3}$ & I & $-\mathrm{CO}_{2} \mathrm{C}_{2} \mathrm{H}_{5}$ & 5 & 81 \\
\hline 5 & $p-\mathrm{COCH}_{3}$ & I & $-\mathrm{C}_{6} \mathrm{H}_{5}$ & 5 & 82 \\
\hline 6 & $p-\mathrm{CO}_{2} \mathrm{C}_{2} \mathrm{H}_{5}$ & I & $-\mathrm{C}_{6} \mathrm{H}_{5}$ & 5 & 85 \\
\hline 7 & $p-\mathrm{OCH}_{3}$ & I & $-\mathrm{C}_{6} \mathrm{H}_{5}$ & 5 & 80 \\
\hline 8 & $p-\mathrm{COCH}_{3}$ & $\mathrm{Br}$ & $-\mathrm{CO}_{2} \mathrm{C}_{2} \mathrm{H}_{5}$ & 10 & 74 \\
\hline 9 & $p-\mathrm{OCH}_{3}$ & $\mathrm{Br}$ & $-\mathrm{CO}_{2} \mathrm{C}_{2} \mathrm{H}_{5}$ & 10 & 72 \\
\hline 10 & $p-\mathrm{COCH}_{3}$ & $\mathrm{Br}$ & $-\mathrm{C}_{6} \mathrm{H}_{5}$ & 10 & 72 \\
\hline 11 & $p-\mathrm{OCH}_{3}$ & $\mathrm{Br}$ & $-\mathrm{C}_{6} \mathrm{H}_{5}$ & 10 & 70 \\
\hline 12 & $o-\mathrm{CO}_{2} \mathrm{CH}_{3}$ & $\mathrm{Br}$ & $-\mathrm{CO}_{2} \mathrm{C}_{2} \mathrm{H}_{5}$ & 10 & 57 \\
\hline 13 & $o-\mathrm{CO}_{2} \mathrm{CH}_{3}$ & $\mathrm{Br}$ & $-\mathrm{C}_{6} \mathrm{H}_{5}$ & 10 & 53 \\
\hline 14 & $\mathrm{H}$ & $\mathrm{Br}$ & $-\mathrm{CO}_{2} \mathrm{C}_{2} \mathrm{H}_{5}$ & 5 & 68 \\
\hline 15 & $\mathrm{H}$ & $\mathrm{Br}$ & $-\mathrm{C}_{6} \mathrm{H}_{5}$ & 5 & 65 \\
\hline 16 & $\mathrm{H}$ & $\mathrm{Br}$ & $-\mathrm{CN}$ & 5 & 69 \\
\hline 17 & $\mathrm{H}$ & $\mathrm{Br}$ & $-\mathrm{CO}_{2}\left(\mathrm{CH}_{2}\right)_{3} \mathrm{CH}_{3}$ & 10 & 65 \\
\hline 18 & $\mathrm{H}$ & $\mathrm{Br}$ & $-\mathrm{CO}_{2} \mathrm{CH}_{2} \mathrm{CH}\left(\mathrm{C}_{2} \mathrm{H}_{5}\right)\left(\mathrm{CH}_{2}\right)_{3} \mathrm{CH}_{3}$ & 10 & 62 \\
\hline 19 & $p-\mathrm{CO}_{2} \mathrm{C}_{2} \mathrm{H}_{5}$ & $\mathrm{Cl}$ & $-\mathrm{CO}_{2} \mathrm{C}_{2} \mathrm{H}_{5}$ & 20 & NR \\
\hline 20 & $p-\mathrm{COCH}_{3}$ & $\mathrm{Cl}$ & $-\mathrm{CO}_{2} \mathrm{C}_{2} \mathrm{H}_{5}$ & 20 & NR \\
\hline
\end{tabular}

$\mathrm{PdCl}_{2}(0.01 \mathrm{mmol})$, ionic liquid (IL-2) $2.0 \mathrm{~mL}$, haloarene $(1 \mathrm{mmol})$, olefin ( $\left.2 \mathrm{mmol}\right)$, Microwave power $200 \mathrm{~W}$, reaction temperature $120^{\circ} \mathrm{C}$. ${ }^{\mathrm{a}}$ The isolated $(E)$-products are confirmed by ${ }^{1} \mathrm{H} \mathrm{NMR}$

Recyclability of ionic liquid (IL-2) was investigated using iodobenzene (1 mmol), ethyl acrylate $(2 \mathrm{mmol})$ in ionic liquid (IL-2) and its results are listed in Table 3. Ionic liquid (IL-2) has showed good isolated yields in the range of $80 \%-84 \%$ for six consecutive runs without significant loss of activity. 
Table 3. Recyclability of the ionic liquid (IL-2) for palladium catalyzed Heck reaction

\begin{tabular}{ccccccc}
\hline \multirow{2}{*}{ Entry } & \multicolumn{7}{c}{ Isolated Yield (\%) } \\
\cline { 2 - 7 } & Run 1 & Run 2 & Run 3 & Run 4 & Run 5 & Run 6 \\
\hline 1 & 84 & 84 & 83 & 83 & 82 & 80 \\
2 & 83 & 82 & 82 & 82 & 80 & 80 \\
\hline
\end{tabular}

$\mathrm{PdCl}_{2}(0.01 \mathrm{mmol})$, ionic liquid (IL-2) $2.0 \mathrm{~mL}$, iodoarene $(1.0 \mathrm{mmol})$, ethyl acrylate $(2.0 \mathrm{mmol})$, Microwave power $200 \mathrm{~W}$, reaction temperature $120^{\circ} \mathrm{C}$, reaction time $5.0 \mathrm{~min}$.

\section{Conclusions}

In conclusion, we have successfully developed an efficient, air stable and phosphine free catalytic system for Heck reaction using ionic liquid (IL-2) as solvent, ligand and base and palladium chloride as a catalyst. The in-situ formed palladium complex proved to be an excellent catalyst for olefination of activated and deactivated iodo- and bromoarenes including sterically hindered, methyl-2-bromo-benzoate. It also exhibited significant efficiency for less reactive vinyl acrylates such as n-butyl acrylate and 2-ethylhexyl acrylate. Due to the advantageous features of the ionic liquid (IL-2) like multiple coordinating sites, the palladium catalyst showed good stability and could be recycled for six consecutive runs without loss of activity. The mechanism for the Heck reaction in ionic liquid (IL-2) in presence of $\mathrm{PdCl}_{2}$ is being experimentally validated. Further investigation of the developed catalytic system for various palladiumcatalyzed coupling reactions is under investigation.

\section{Experimental Section}

General. The ionic liquid was characterized by FTIR, ${ }^{1} \mathrm{HNMR}$, Mass spectroscopy and CHN analysis. FTIR spectra were obtained on a Shimadzu FTIR-8400S spectrometer using neat samples and ${ }^{1} \mathrm{H}$ NMR $(300 \mathrm{MHz})$ and ${ }^{13} \mathrm{C}$ NMR $(75 \mathrm{MHz})$ data were recorded on a Varian Mercury VXR-300 NMR spectrometer using DMSO and $\mathrm{CDCl}_{3}$ as a solvent with an internal standard. ESI-MS was obtained with micromass - Q - Tof (YA105) spectrometer and elemental analysis (C,H,N elements) were carried out using ThermoFinnigan EA112 Elemental analyser. All reactions were carried out in CEM Discover Benchmate ${ }^{\mathrm{TM}}$ microwave appratus (CEM Corp.). All chemicals were purchased from Sigma Aldrich, S.D. Fine chemicals and Spectrochem private Ltd, India and were used without further purification.

1-(2-Cyanoethyl)-3-(2-hydroxyethyl)-1H-imidazol-3-ium tetrafluoroborate (IL-2). In this procedure, $1 \mathrm{H}$-imidazole $(25 \mathrm{mmol})$ was dissolved in anhydrous methanol and acrylonitrile $(25$ mmol) was added to the resultant solution. The reaction mixture was heated to $70{ }^{\circ} \mathrm{C}$ for $24 \mathrm{~h}$, 
and then 2-chloroethanol $(25 \mathrm{mmol})$ was added to the reaction mixture and stirred at $70{ }^{\circ} \mathrm{C}$ for another $48 \mathrm{~h}$. The reaction was cooled to room temperature and $\mathrm{NaBF}_{4}(30 \mathrm{mmol})$ was added to the reaction mixture, stirred at room temperature for $48 \mathrm{~h}$, then filter and solution was concentrated under vacuum to get brown red viscous liquid (Yield $=82 \%$ ). IR (neat, $\mathrm{cm}^{-1}$ ): 3559 $(\mathrm{OH}), 2252(\mathrm{CN}) .{ }^{1} \mathrm{H}$ NMR $\left(300 \mathrm{MHz}, \mathrm{DMSO}-d_{6}, \mathrm{ppm}\right): \delta_{\mathrm{H}} 9.15$ (s, $\left.1 \mathrm{H}, \mathrm{NC}(\mathrm{H}) \mathrm{N}^{+}\right), 7.75(\mathrm{dd}$, $\left.2 \mathrm{H}, J=9.3 \mathrm{~Hz}, \mathrm{NC}(\mathrm{H}) \mathrm{C}(\mathrm{H}) \mathrm{N}^{+}\right), 5.21(\mathrm{~s}, 1 \mathrm{H}, \mathrm{OH}), 4.50\left(\mathrm{t}, 2 \mathrm{H}, J=13.1 \mathrm{~Hz}, \mathrm{CH}_{2} \mathrm{~N}\right), 4.24(\mathrm{t}, 2 \mathrm{H}, J$ $\left.=9.6 \mathrm{~Hz}, \mathrm{CH}_{2} \mathrm{~N}^{+}\right), 3.73\left(\mathrm{t}, 2 \mathrm{H}, J=9.9 \mathrm{~Hz}, \mathrm{OH}-\mathrm{CH}_{2}\right), 3.17\left(\mathrm{t}, 2 \mathrm{H}, J=12.9 \mathrm{~Hz}, \mathrm{CH}_{2}-\mathrm{CN}\right) .{ }^{13} \mathrm{C}$ NMR (75 MHz DMSO-d $\left.d_{6}\right): \delta=137.41\left(\mathrm{NC}_{\mathrm{Ar}} \mathrm{N}\right), 123.81\left(\mathrm{NCH}_{\mathrm{Ar}} \mathrm{CH}_{\mathrm{Ar}}\right), 122.81\left(\mathrm{NCH}_{\mathrm{Ar}} \mathrm{CH}_{\mathrm{Ar}}\right)$, $118.32(\mathrm{CHCN}), 59.87(\mathrm{CHOH}), 52.54\left(\mathrm{~N}_{\mathrm{Ar}} \mathrm{CHCHOH}\right), 45.04\left(\mathrm{~N}_{\mathrm{Ar}} \mathrm{CHCHCN}\right), 19.32(C \mathrm{HCN})$ ppm. ESI-MS: $M^{+} / z$ 166, $M^{-} / z$ 87. Elemental Analysis: $\mathrm{C}_{8} \mathrm{H}_{12} \mathrm{BF}_{4} \mathrm{~N}_{3} \mathrm{O}$, calcd (\%): C 37.98, $\mathrm{H}$ 4.78, N 16.61; found: C 37.939, H 4.771, N 16.677.

\section{General procedure for palladium catalyzed Heck reaction. (E)-Acetyl stilbene (5)}

In a typical example, 4-iodo-acetophenone $(1 \mathrm{mmol})$, styrene $(2 \mathrm{mmol})$ and $\mathrm{PdCl}_{2}(0.01 \mathrm{mmol})$ were added to $2 \mathrm{~mL}$ of ionic liquid (IL-2) in a microwave vessel. Vessel was then sealed and kept in the microwave cavity (CEM Discover Benchmate ${ }^{\mathrm{TM}}$, CEM Corp). Initially microwave irradiation of 200 Watt was applied to reach the target temperature of $120^{\circ} \mathrm{C}$ (measured using built-in IR temperature device); the reaction mixture was stirred at this temperature for $5 \mathrm{~min}$. During this time, the microwave power was varied automatically to retain the target temperature at $120{ }^{\circ} \mathrm{C}$. The reaction mixture was allowed to cool to room temperature. The product was extracted with diethyl ether and ether layer was concentrated under reduced pressure. The residue was purified by column chromatography using hexane: ethyl acetate (9:1) as a solvent, to obtain $(E)$-acetyl stilbene as white solid (Yield = 82\%). mp $149-150{ }^{\circ} \mathrm{C}\left(\mathrm{Lit}^{23}{ }^{23}\right.$ 150-151 $\left.{ }^{\circ} \mathrm{C}\right) ;{ }^{1} \mathrm{H}-$ NMR (300 MHz, $\left.\mathrm{CDCl}_{3}, \mathrm{Me}_{4} \mathrm{Si}, \mathrm{ppm}\right): \delta 7.91(2 \mathrm{H}, \mathrm{d}, J 12 \mathrm{~Hz}), 7.64(4 \mathrm{H}, \mathrm{m}, J 21.0 \mathrm{~Hz}), 7.42$ $(2 \mathrm{H}, \mathrm{dd}, J 9.0 \mathrm{~Hz}), 7.35(1 \mathrm{H}, \mathrm{dd}, J 11.0 \mathrm{~Hz}), 7.23(2 \mathrm{H}, \mathrm{dd}, J 15.0 \mathrm{~Hz}), 2.66(3 \mathrm{H}, \mathrm{s}) .{ }^{13} \mathrm{C} \mathrm{NMR}(75$ $\left.\mathrm{MHz} \mathrm{CDCl}_{3}\right): \delta=197.68(\mathrm{CHCOCH}), 142.25\left(\mathrm{CHC} \mathrm{Ar}_{\mathrm{AH}}\right), 136.93\left(\mathrm{CHC}_{\mathrm{Ar}} \mathrm{CH}\right), 136.20$ $\left(\mathrm{CHC} C_{\mathrm{Ar}} \mathrm{CH}\right), 131.70\left(C_{\mathrm{Ar}}\right), 129.10\left(\mathrm{CH}_{\mathrm{Ar}}\right), 129.02\left(\mathrm{CH}_{\mathrm{Ar}}\right), 128.54\left(\mathrm{CH}_{\mathrm{Ar}}\right), 127.68\left(C \mathrm{H}_{\mathrm{Ar}}\right)$, $127.04\left(\mathrm{CH}_{\mathrm{Ar}}\right), 126.73\left(\mathrm{CH}_{\mathrm{Ar}}\right), 26.79(\mathrm{COCH})$ ppm. Elemental Analysis: $\mathrm{C}_{16} \mathrm{H}_{14} \mathrm{O}$, calcd $(\%)$ : $\mathrm{C}$ 86.45, H 6.35; found: C 86.459, H 6.354.

(E)-Ethyl cinnamate (2). Colorless oil ${ }^{23}{ }^{1} \mathrm{H}-\mathrm{NMR}\left(300 \mathrm{MHz}, \mathrm{CDCl}_{3}, \mathrm{Me}_{4} \mathrm{Si}, \mathrm{ppm}\right)$ : $\delta 7.71(1 \mathrm{H}$, d, $J 16.0 \mathrm{~Hz}), 7.53-7.56(2 \mathrm{H}, \mathrm{m}), 7.39-7.41(3 \mathrm{H}, \mathrm{m}), 6.46(1 \mathrm{H}, \mathrm{d}, J 16.0 \mathrm{~Hz}), 4.29(2 \mathrm{H}, \mathrm{q}, J$ $7.1 \mathrm{~Hz}), 1.36(3 \mathrm{H}, \mathrm{t}, J 7.1 \mathrm{~Hz})$.

(E)-Ethyl 3-(4-acetylphenyl) acrylate (1). White crystals; mp $41-42{ }^{\circ} \mathrm{C}$ ( Lit. $\left.^{19(a)} 42-43{ }^{\circ} \mathrm{C}\right) ;{ }^{1} \mathrm{H}-$ NMR (300 MHz, $\left.\mathrm{CDCl}_{3}, \mathrm{Me}_{4} \mathrm{Si}, \mathrm{ppm}\right): \delta 7.91(2 \mathrm{H}, \mathrm{d}, J 8.0 \mathrm{~Hz}), 7.73(1 \mathrm{H}, \mathrm{d}, J 16 \mathrm{~Hz}), 7.62(2 \mathrm{H}$, d, $J 1.5 \mathrm{~Hz}), 6.52(1 \mathrm{H}, \mathrm{d}, J 16.0 \mathrm{~Hz}), 4.35(2 \mathrm{H}, \mathrm{q}, J 21.0 \mathrm{~Hz}), 2.67(3 \mathrm{H}, \mathrm{s}), 1.35(3 \mathrm{H}, \mathrm{t}, J 14.0 \mathrm{~Hz})$.

(E)-Ethyl 4-(3-ethoxy-3-oxoprop-1-enyl) benzoate (3). White solid; mp 51-52 ${ }^{\circ} \mathrm{C}$ (Lit. $^{22} 51-52$ $\left.{ }^{\circ} \mathrm{C}\right) ;{ }^{1} \mathrm{H}-\mathrm{NMR}\left(300 \mathrm{MHz}, \mathrm{CDCl}_{3}, \mathrm{Me}_{4} \mathrm{Si}, \mathrm{ppm}\right): \delta 8.05(2 \mathrm{H}, \mathrm{d}, J 8.6 \mathrm{~Hz}), 7.70(1 \mathrm{H}, \mathrm{d}, J 16.0 \mathrm{~Hz})$, $7.58(2 \mathrm{H}, \mathrm{d}, J 8.6 \mathrm{~Hz}), 6.52(1 \mathrm{H}, \mathrm{d}, J 16.0 \mathrm{~Hz}), 4.39(2 \mathrm{H}, \mathrm{q}, J 7.0 \mathrm{~Hz}), 4.28(2 \mathrm{H}, \mathrm{q}, J 7.2 \mathrm{~Hz}), 1.40$ $(3 \mathrm{H}, \mathrm{t}, J 7.0 \mathrm{~Hz}), 1.35(3 \mathrm{H}, \mathrm{t}, J 7.2 \mathrm{~Hz})$. 
(E)-Ethyl 4-methoxy cinnamate (4). White crystals; mp 49-50 ${ }^{\circ} \mathrm{C}$ (Lit. $\left.{ }^{19(a)} 50-51{ }^{\circ} \mathrm{C}\right) ;{ }^{1} \mathrm{H}-\mathrm{NMR}$ (300 MHz, $\left.\mathrm{CDCl}_{3}, \mathrm{Me}_{4} \mathrm{Si}, \mathrm{ppm}\right): \delta 7.64(1 \mathrm{H}, \mathrm{d}, J 15.9 \mathrm{~Hz}), 7.46(2 \mathrm{H}, \mathrm{d}, J 9.0 \mathrm{~Hz}), 6.88(2 \mathrm{H}, \mathrm{d}, J$ $9.0 \mathrm{~Hz}), 6.30(1 \mathrm{H}, \mathrm{d}, J 15.9 \mathrm{~Hz}), 4.24(2 \mathrm{H}, \mathrm{q}, J 7.2 \mathrm{~Hz}), 3.81(3 \mathrm{H}, \mathrm{s}), 1.32(3 \mathrm{H}, \mathrm{t}, J 7.2 \mathrm{~Hz})$.

(E)-Ethyl-4-styrylbenzoate (6). White solid; mp 107-108 ${ }^{\circ} \mathrm{C}$ (Lit. ${ }^{23}$ 107-108 $\left.{ }^{\circ} \mathrm{C}\right) ;{ }^{1} \mathrm{H}-\mathrm{NMR}(300$ $\left.\mathrm{MHz}, \mathrm{CDCl}_{3}, \mathrm{Me}_{4} \mathrm{Si}, \mathrm{ppm}\right): \delta 8.04(2 \mathrm{H}, \mathrm{d}, J 8.4 \mathrm{~Hz}), 7.55(4 \mathrm{H}, \mathrm{m}), 7.41(2 \mathrm{H}, \mathrm{m}), 7.32(1 \mathrm{H}, \mathrm{m})$, $7.22(1 \mathrm{H}, \mathrm{d}, J 16.3 \mathrm{~Hz}), 7.12(1 \mathrm{H}, \mathrm{d}, J 16.3 \mathrm{~Hz}), 4.39(2 \mathrm{H}, \mathrm{q}, J 7.1 \mathrm{~Hz}), 1.41(3 \mathrm{H}, \mathrm{t}, J 7.1 \mathrm{~Hz})$.

(E)-4-Methoxystilbene (7). White solid, mp 136-137 ${ }^{\circ} \mathrm{C}$ (Lit. ${ }^{23}$ 138-139 ${ }^{\circ} \mathrm{C}$ ); ${ }^{1} \mathrm{H}-\mathrm{NMR}(300$ $\left.\mathrm{MHz}, \mathrm{CDCl}_{3}, \mathrm{Me}_{4} \mathrm{Si}, \mathrm{ppm}\right): \delta 7.51(4 \mathrm{H}, \mathrm{m}), 7.38(2 \mathrm{H}, \mathrm{m}), 7.24(1 \mathrm{H}, \mathrm{m}), 7.08(1 \mathrm{H}, \mathrm{d}, J 16.3 \mathrm{~Hz})$, $6.94(1 \mathrm{H}, \mathrm{d}, J 16.3 \mathrm{~Hz}), 6.90(2 \mathrm{H}, \mathrm{d}, J 7.5 \mathrm{~Hz}), 3.84(3 \mathrm{H}, \mathrm{s})$.

(E)-Methyl 2-(3-ethoxy-3-oxoprop-1-enyl) benzoate (12). Colorless oil; ${ }^{24} \mathrm{H}-\mathrm{NMR}(300 \mathrm{MHz}$, $\left.\mathrm{CDCl}_{3}, \mathrm{Me}_{4} \mathrm{Si}, \mathrm{ppm}\right): \delta 8.05(2 \mathrm{H}, \mathrm{d}, J 8.7 \mathrm{~Hz}), 7.70(1 \mathrm{H}, \mathrm{d}, J 15.9 \mathrm{~Hz}), 7.59(2 \mathrm{H}, \mathrm{d}, J 8.7 \mathrm{~Hz}), 6.52$ $(1 \mathrm{H}, \mathrm{d}, J 15.9 \mathrm{~Hz}), 4.29(2 \mathrm{H}, \mathrm{q}, J 7.2 \mathrm{~Hz}), 3.94(3 \mathrm{H}, \mathrm{s}), 1.35(3 \mathrm{H}, \mathrm{t}, J 7.2 \mathrm{~Hz})$.

(E)-Methyl 2-styrylbenzoate (13). Yellow oil; ${ }^{25} \mathrm{H}-\mathrm{NMR}\left(300 \mathrm{MHz}, \mathrm{CDCl}_{3}, \mathrm{Me}_{4} \mathrm{Si}, \mathrm{ppm}\right)$ : $\delta$ $7.97(10 \mathrm{H}, \mathrm{m}), 7.21(1 \mathrm{H}, \mathrm{d}, J 16.0 \mathrm{~Hz}), 6.90(1 \mathrm{H}, \mathrm{d}, J 16.0 \mathrm{~Hz}), 4.30(3 \mathrm{H}, \mathrm{s})$.

(E)-Stilbene (15). White solid; mp 121-122 ${ }^{\circ} \mathrm{C}$ (Lit. ${ }^{23}$ 122-123 $\left.{ }^{\circ} \mathrm{C}\right) ;{ }^{1} \mathrm{H}-\mathrm{NMR}\left(300 \mathrm{MHz}, \mathrm{CDCl}_{3}\right.$, $\left.\mathrm{Me}_{4} \mathrm{Si}, \mathrm{ppm}\right): \delta 7.15(2 \mathrm{H}, \mathrm{s}), 7.30(2 \mathrm{H}, \mathrm{m}), 7.39$ (4H, m), $7.56(4 \mathrm{H}, \mathrm{m})$.

(E)-Cinnamonitrile (16). Colorless liquid; ${ }^{26}{ }^{1} \mathrm{H}-\mathrm{NMR}$ (300 MHz, $\mathrm{CDCl}_{3}, \mathrm{Me}_{4} \mathrm{Si}, \mathrm{ppm}$ ): $\delta 5.87$ $(1 \mathrm{H}, \mathrm{d}, J 16.7 \mathrm{~Hz}), 7.41(1 \mathrm{H}, \mathrm{d}, J 16.7 \mathrm{~Hz}), 7.46(5 \mathrm{H}, \mathrm{m})$.

(E)-n-Butyl cinnamate (17). Yellow liquid; ${ }^{27} \mathrm{H}-\mathrm{NMR}\left(300 \mathrm{MHz}, \mathrm{CDCl}_{3}, \mathrm{Me}_{4} \mathrm{Si}, \mathrm{ppm}\right): \delta 7.68$ $(1 \mathrm{H}, \mathrm{d}, J 15.9 \mathrm{~Hz}), 7.53(2 \mathrm{H}, \mathrm{m}), 7.50(2 \mathrm{H}, \mathrm{m}) 7.38(3 \mathrm{H}, \mathrm{m}), 6.44(1 \mathrm{H}, \mathrm{d}, J 15.9 \mathrm{~Hz}), 4.21(2 \mathrm{H}, \mathrm{t}$, $J 7.2 \mathrm{~Hz}), 1.74(2 \mathrm{H}, \mathrm{m}), 1.37(2 \mathrm{H}, \mathrm{m}), 0.96(3 \mathrm{H}, \mathrm{t}, J 7.2 \mathrm{~Hz})$.

(E)-2-Ethylhexyl cinnamate (18). Colorless liquid; ${ }^{28}{ }^{1} \mathrm{H}-\mathrm{NMR}\left(300 \mathrm{MHz}, \mathrm{CDCl}_{3}, \mathrm{Me}_{4} \mathrm{Si}, \mathrm{ppm}\right)$ : $\delta 7.68(1 \mathrm{H}, \mathrm{d}, J 16.0 \mathrm{~Hz}), 7.54(2 \mathrm{H}, \mathrm{m}), 7.39(3 \mathrm{H}, \mathrm{m}), 6.45(1 \mathrm{H}, \mathrm{d}, J 16.0 \mathrm{~Hz}), 4.16(2 \mathrm{H}, \mathrm{m}), 1.66$ $(1 \mathrm{H}, \mathrm{m}), 1.44(8 \mathrm{H}, \mathrm{m}), 0.89-0.95(6 \mathrm{H}, \mathrm{m})$.

\section{General procedure for recyclability of catalytic system}

After extracting the mixture from diethyl ether, Ionic liquid (IL-2) was dissolved in acetone (5 $\mathrm{mL}$ ). Then, neutralization of the acid generated during the reaction was done by addition of the potassium carbonate. The salt precipitated out was filtered off and acetone layer was concentrated under reduced pressure at room temperature. Without any further purification; the resultant residue was used for the next run, after addition of iodobenzene (1 mmol), ethyl acrylate $(2 \mathrm{mmol})$ again, for six consecutive runs.

\section{Acknowledgements}

I am thankful to University Grant Commission, Delhi for financial support for project and fellowship and also Omkar Chemicals, Mumbai for providing useful chemicals. 


\section{References}

1. (a) Beletskaya, I. P.; Cheprakov, A. V. Chem. Rev. 2000, 100, 3009. (b) Priebbenow, D. L.; Stewart, S. G.; Pfeffer, F. M. Org. Biomol. Chem. 2011, 9, 1508. (c) Wang, D.; Yu, J. J. Am. Chem. Soc. 2011, 133, 5767. (d) Gottumukkala, A. L.; De Varies, J. G.; Minnaard, A. J. Chem. Eur. J. 2011, 17, 3091.

2. Ritter, K. Synthesis 1993, 735.

3. Blaser, H. U.; Spencer, A. J. Organomet. Chem. 1982, 233, 267.

4. Stephan, M. S.; Teunissen, A. J. J. M.; Verzijl, G. K. M.; De Vries, J. G. Angew. Chem. Int. Ed. Engl. 1998, 37, 662.

5. Miura, M.; Hashimoto, H.; Itoh, K.; Nomura, M. J. Chem. Soc. Perkin Trans. 1990, 1, 2207.

6. (a) Kikukawa, K.; Matsuda, T. Chem. Lett. 1977, 159. (b) Cacchi, S.; Fabrizi G.; Goggiamani, A.; Sferrazza, A. Org. Biomol. Chem. 2011, 9, 1727.

7. De Vries, J. G. J. Can. Chem. 2001, 79, 1086.

8. Yang, C.; Lee, H. M.; Nolan, S. P. Org. Lett. 2001, 3, 1511.

9. (a) Consorti, C.S.; Flores, F.R.; Dupont, J. J. Am. Chem. Soc. 2005, 127, 12054. (b) Bergbreier, D. E.; Osburn, P. L.; Wilson, A.; Sink, E. J. Am. Chem. Soc. 2000, 122, 9058. (c) Calo, V.; Nacci, A.; Lopez, L.; Maarini, N. Tetrahedron Lett. 2000, 41, 8973. (d) Wang, R.; Twamley, B.; Shreeve, J. M. J. Org. Chem. 2006, 71, 426. (e) Li, H. J.; Wang, L. Eur. J. Org. Chem. 2006, 5099. (f) Kwong, F. Y.; Klapars, A.; Buckwald, S. I. Org. Lett. 2002, 4, 581. (g) Gottumukkala, A. L. ; Teichert, J. F.; Heijnen, D.; Eisink, N.; Dijk, S. V.; Ferrer, C.; Hoogenband, A. V. D. ; Minnaard, A. J. J. Org. Chem. 2011, 76, 3498.

10. Cui, X.; Zhou, Y.; Wang, N.; Liu, L.; Guo, Q. X. Tetrahedron Lett. 2007, 48, 163.

11. (a) Reetz, M. T.; de Vries, J. G. Chem. Commun. 2004, 1559. (b) Yao, Q.-W.; Kinney, E. P.; Yang, Z. J. Org. Chem. 2003, 68, 7528. (c) Jeffery, T. Tetrahedron 1996, 52, 10113. (d) de Vries, A. H. M.; Mulders, J. M. C. A.; Mommers, J. H. M.; Henelerickx, H. J. W.; de Vries, J. G. Org. Lett. 2003, 5, 3285. (e) Chandrasekhar, S.; Narsihmulu, C.; Sultana, S. S.; Reddy, N. R. Org. Lett. 2002, 4, 4399. (f) Gozzi, C.; Lavenot, L.; Ilg, K.; Penalva, V.; Lemaire, M.

12. Tetrahedron Lett. 1997, 38, 8867.

13. (a) Ramchandani, R. K.; Uphade, B. S.; Vinod, M. P.; Wakharkar, R. D.; Choudhary, V. R.; Sudalai, A. Chem. Commun. 1997, 2071. (b) Mehnert, C. P.; Weaver, D. W.; Ying, J. K. J. Am. Chem. Soc. 1998, 120, 12289. (c) Anson, M. S.; Mirza, A. R.; Tonks, L.; Williams, J. M. J. Tetrahedron Lett. 1999, 40, 7147. (d) Zhao, F.-Y.; Bhanage, B. M.; Shirai, M.; Arai, M. Chem.-Eur. J. 2000, 6, 843. (e) Djakovitch, L.; Koehler, K. J. Am. Chem. Soc. 2001, 123, 5990. (f) Mori, K.; Yamaguchi, K.; Hara, T.; Mizugaki, T.; Ebitani, K.; Kaneda, K. J. Am. Chem. Soc. 2002, 124, 11572. (g) Biffis, A.; Zecca, M.; Basato, M. J. Mol. Catal. A: Chem. 2001, 173, 249. (h) Blaser, H. U.; Indolese, A.; Schnyder, A.; Steiner, H.; Studer, M. J. Mol. Catal. A: Chem. 2001, 173, 3. (i) Molnar, A.; Papp, A.; Miklos, K.; Forgo, P. Chem. Commun. 2003, 2626. (j) Prockl, S. S.; Kleist, W.; Gruber, M. A.; Kohler, K. Angew. Chem., Int. Ed. 2004, 43, 1881. 
14. (a) Djakovitch, L.; Koehler, K. J. Mol. Catal. A: Chem. 1999, 142, 275. (b) Kohler, K.; Heidenreich, R. G.; Krauter, J. G. E.; Pietsch, J. Chem.-Eur. J. 2002, 8, 622. (c) Okumura, K.; Tomiyama, T.; Moriyama, S.; Nakamichi, A.; Niwa, M. Molecules 2011, 16, 38.

15. (a) Dupont, J.; De Souza, R. F.; Saurez, P. A. Chem. Rev. 2002, 102, 3667. (b) Welton, T. Chem. Rev. 1999, 99, 2071. (c) Earle, M. J.; Seddon, K. R. Pure App. Chem. 2000, 72, 1391.

(d) Gyton, M. R.; Cole, M. L.; Harper, J. B. Chem. Commun. 2011, 47, 9200.

16. (a) Pei, W.; Mo, J.; Xiao, J. J. Organomet. Chem. 2005, 690, 3546. (b) Mo, J.; Xu, L.; Ruan, J.; Liu, S.; Xiao, J. Chem. Comm. 2006, 3591.

17. Bellina, F.; Chiappe, C. Molecules 2010, 15, 2211.

18. (a) Cai, Y. Q.; Lu, Y.; Liu, Y.; Gao, G. H. Catal. Lett. 2007, 119, 154. (b) Cai, Y.; Liu, Y. Cat. Commun. 2009, 10, 1390.

19. Youquan, Z. D. China Patent, CN1978433A, 2007.

20. (a) Shaaban, M. R.; Darweesh, A. F.; Dawood, K. M.; Farag, A. M. Arkivoc 2010, (x), 208.

(b) Dawood, K. M.; El-Deftar, M. M. Arkivoc 2010, (ix), 319.

21. Xie, X.; Lu, J.; Chen, B.; Han, J.; She, X.; Pan X. Tetrahedron Lett. 2004, 45, 809.

22. (a) Vallin, K. S. A.; Emilsson, P.; Larhed, M.; Hallberg, A. J. Org. Chem. 2002, 67, 6243.

(b) Choudary, B. M.; Madhi, S.; Chowdari, N. S.; Kantam, M. L.; Sreedhar, B. J. Am. Chem. Soc. 2002, 124, 14127.

23. Huang, S. H.; Chen, J. R.; Tsai, F. Y. Molecules 2010, 15, 315.

24. Wang, L.; Li, H.; Li, P. Tetrahedron 2009, 65, 364.

25. Borhade, S. R.; Waghmode, S. B. Tetrahedron Lett. 2008, 49, 3423.

26. Shahzad, S. A.; Vivant, C.; Wirth, T. Org. Lett. 2010, 12, 1364.

27. Yamaguchi, K.; Fujiwara, H.; Ogasawara, Y.; Kotani, M.; Mizuno, N. Angew. Chem. Int. Ed. Engl. 2007, 46, 3922.

28. Andappan, M. M. S.; Nilsson, P.; Larhed, M. Chem. Commun. 2004, 2, 218.

29. Reetz, M. T.; Westermann, E.; Lohmer, R.; Lohmer, G. Tetrahedron Lett. 1998, 39, 8449. 